\title{
Inventory-Routing Model, for a multi-period problem with stochastic and deterministic demand.
}

\author{
Rita Ribeiro* Helena R. Lourenço ${ }^{\dagger}$
}

\begin{abstract}
The need for integration in the supply chain management leads us to consider the coordination of two logistic planning functions: transportation and inventory. The coordination of these activities can be an extremely important source of competitive advantage in the supply chain management. The battle for cost reduction can pass through the equilibrium of transportation versus inventory managing costs. In this work, we study the specific case of an inventory-routing problem for a week planning period with different types of demand. A heuristic methodology, based on the Iterated Local Search, is proposed to solve the Multi-Period Inventory Routing Problem with stochastic and deterministic demand.

Keywords: Inventory-Routing, Iterated Local Search, Logistics.

JEL: C61, C63, M19
\end{abstract}

\section{Introduction}

In many industries, the logistic planning functions of transportation and inventory play an important role and integrating these two areas may lead to significant gains and more competitive distribution strategies. The coordination of these activities can be an extremely important source of competitive advantage in the supply chain management. The battle for cost reduction

*Department of Economics and Business and GREL-IET, Universitat Pompeu Fabra, Ramon Trias Fargas 25-27, 08005 Barcelona (Spain). Tel: 34-93 5422692, Fax: 34-93 5421746. E-mail: rita.ribeiro@upf.edu. This research has been partially financed by research grant from Ministério da Ciência e a Tecnologia, FCT, Praxis XXI/BD/21814/99.

${ }^{\dagger}$ Department of Economics and Business and GREL-IET, Universitat Pompeu Fabra, Ramon Trias Fargas 25-27, 08005 Barcelona (Spain). Tel: 34-93 5422753, Fax: 34-93 5421746. E-mail: helena.ramalhinho@upf.edu. This research has been partially financed by research grant MCYT BEC-2000 and GREL-IET. 
can pass through the equilibrium of transportation costs versus inventory managing costs.

Both the Vehicle Routing (VR) and the Inventory Management (IM) problems have been extensively studied separately and there exists a vast amount of literature on these areas. However, when looking at the two problems together, the amount of work found is much less. Many models have been proposed for inventory problems with no routing decisions considered and many studies exists on vehicle routing problems in which no inventory management is mentioned.

The problem that considers VR and IM decisions together is known as the Inventory Routing Problem (IRP). The main objective in the IRP is to design the set of routes and delivery quantities that minimize transportation cost while controlling inventory costs. Considering these two problems in an integrated manner can reduce total costs.

The model we propose is a Multi-Period Inventory-Routing with two types of customers: the Vendor-Managed Inventory (VMI) customers and the Customer Managed Inventory (CMI) customers. The VMI customers have a random demand and the distributor manages the stock at the VMI customer location. The CMI have fixed demand and the distributor faces no inventory costs associated with these customers.

The objective is to determine the routes for a week planning period and the quantities delivered to the VMI points, minimizing total transportation plus inventory costs.

The motivation of this work can be found on the advantages that can be obtained when integrating processes along the supply chain. In this particular case, trying to reduce total costs through the coordination of decisions between distribution management and inventory management areas. Although there exist some literature on the integration issue, none of them addresses the specific case of this model: two types of customers, a week minimum visit and no information on the inventory levels during the period.

This paper is organized in the following way: First we make a review on some relevant IRP literature. Then, we expose our Multi-Period Inventory-Routing Problem with Stochastic and Deterministic Demand (MPIRP-SDD) model in detail. In section 4, the solution method, based on the Iterated Local Search is presented. In section 5, we show the results of a computational experiment. And finally, we draw some conclusions and further research. 


\section{Literature review}

Ferdergruen and Simchi-Levi (1995) make a good summary on inventoryrouting problems. These authors divided the IRP models into two variants: the single period model and the infinite horizon model.

Baita et al. (1998) also present a review on dynamic routing and inventory. These problems are characterized by having a dynamic environment. Repeated decisions have to be taken at different times within some time horizon and, earlier decisions influence later decisions.

We will now present a review on IRP literature relevant to our study, separating the existing work into Finite and Infinite Horizon Models.

\section{$2.1 \quad$ Finite Horizon}

For the single period inventory routing model, Ferdergruen and Zipkin (1984) addressed the problem of allocating a scarce resource, available at the central depot, among several locations, each with random demand while planning the deliveries using a fleet of vehicles. At the beginning of the period the initial inventory is reported to the depot. This information is used to determine the allocation of the available product, for the next day, among the locations. At the same time the assignment of customers to vehicles and their routes are determined. After deliveries are made, the demands occur and inventory carrying and shortage costs are incurred at each location proportional to the end of the period inventory level. In this model it is possible to choose not to visit some of the locations.

Dror and Ball (1987), decompose the multi-period problem into series of single period problem. They studied the case where, in each time interval, only customers, who will reach their safety stock level during this interval, are serviced. Trudeau and Dror (1992) solve the problem for stochastic demand.

Bard, Huang, Jaillet and Dror (1998) present a decomposition scheme for solving inventory routing problems with satellite facilities, in which a central depot must restock a subset of customers on an intermittent basis. In this setting, the customer demand is not known with certainty. A unique aspect is the presence of satellite facilities where vehicles can be reloaded and customer deliveries continue until the closing time is reached. Jaillet et al. (2002) present an incremental cost approximation to be used in a rolling horizon framework for the above problem of minimizing total expected annual delivery costs.

Campbell (1999) presents an inventory-routing problem and an opti- 
mization based approach for its solution. In the $1^{\text {st }}$ phase they decide which customers receive a delivery on each day of the planning period and decide on the size of deliveries and on the $2^{\text {nd }}$ phase the actual delivery routes and schedules for each day. They consider a small set of delivery routes, constructed using a cluster and vehicles are allowed to make multiple trips per day. The objective is to minimize the average daily distribution cost during the planning period without causing stockouts. There is no inventory holding costs or stockouts costs included in this model.

In the literature we find IRP with specific characteristics, such as considering a limited amount of product at the warehouse, see Chien et al. (1989). They address the problem of distributing a limited amount of inventory among customers using a fleet of vehicles so as to maximize profit. The problem is to decide how to allocate its available inventory to the different customers and they assume that the warehouse does not have enough supply to satisfy each customer maximum demand. In order to determine which customers must be served and the amount to supply to each selected customer, we need routing cost information so that the marginal profit (revenue minus delivery cost) for each customer can be accurately computed. The delivery cost for each customer depends on the vehicle routes, which in turn requires information about customer selection and the mount of inventory allocated.

Some research can also be found on the Direct Deliveries Strategies ${ }^{1}$ in IRPs. Burns et al. (1985) developed an analytical method for minimizing the costs of distributing freight by truck from a supplier to many customers. They derive formulas for transportation and inventory costs, and determine the optimal trade-off between these costs. The paper analyses and compares two distribution strategies Direct Shipping (Direct Delivery) and Peddling 2 . For Direct Shipping, the optimal shipment size is given by EOQ model while for Peddling, the optimal shipment size is a full truck. There is no VRP, they consider a Minimum Path model inside each region.

Bertazzi et al. (2002) study a multi-period model with deterministic demand in which a set of products is shipped from a common supplier to several retailers. A retailer can be visited several times during the time horizon. A shipping policy consists of determining for each delivery time instant the set of retailers to visit, the quantity of each product to ship to each retailer and the route of the vehicle. The inventory policy is an Order

\footnotetext{
${ }^{1}$ Direct Delivery Strategies consists in shipping separate loads for each customer.

${ }^{2} \mathrm{~A}$ peddling strategy consists in dispatching trucks that deliver items to more than one customer.
} 
Up-to Level policy ${ }^{3}$. They investigate the case of a single product and a single vehicle.

\subsection{Infinite Horizon}

In the Infinite Horizon IRP, Anily and Ferdergruen (1990) developed a model of a one warehouse multi-retailer system with vehicle routing costs. The objective is to determine the feasible replenishment strategies minimizing long run average transportation and inventory costs. A replenishment strategy specifies a collection of regions covering all outlets. If an outlet belongs to several regions a specific fraction of its sales is assigned to each of these regions. Each time, one of the outlets in a given region, receives a delivery; this delivery is made by a vehicle that visits all other outlets in the region as well (in an efficient sequence or route). These authors allow regions to overlap. See Hall (1991) and Anily and Ferdergruen (1991) for more comments on this model.

Anily, S. and A. Federgruen (1993) extended their analysis on the one warehouse multiple retailer system with vehicle routing costs, to the case where inventories may be kept in the warehouse.

Another work on the Infinite Horizon IRP is the one by Barnes-Schuster and Bassok (1997). These authors studied the situation where retailers face random demands from known distributions functions. Ordered goods arrive at depot and are allocated and delivered to the retailers. Retailers see demands, report to the depot and are charged inventory holding and shortage costs; depot decides whether or not to place the order for retailer $i$. The question that the authors answer is: when it will be effective for the depot to use Direct Shipping and Order Up-to Level rounded to full trucks as its sole ordering policy. Effectiveness is defined as the ratio of the long run average cost per period of the policy at hand to a lower bound on the long run average cost over all possible policies.

Chan et al. (1998), consider a distribution system consisting of a single warehouse and many geographically dispersed retailers. The objective is to determine an inventory policy and a routing strategy such that each retailer can meet its demands and the long-run average transportation and inventory costs are minimized. This paper studies the Zero-Inventory Ordering policy ${ }^{4}$

\footnotetext{
${ }^{3} \mathrm{~A}$ Order Up-to Level policy is a policy where every time a retailer is visited, the quantity of each product delivered is such that the maximum level of inventory is reached.

${ }^{4}$ Zero Inventory Policies, are policies under which a retailer is replenished if and only if its inventory is down to zero.
} 
and the Fixed Partition Policies 5 .

Also, Kleyweght et al. (2002), studied the problem of determining optimal policies for the distribution of a single product from a single supplier to multiple customers. The objective is to maximize expected discounted value, incorporating sales revenues, production costs, transportation costs, inventory holding costs and shortage penalties, over an infinite horizon. They study the special case of the Direct Deliveries.

In Berman and Larson (2001), the objective is to adjust dynamically the amount of product provided on scene to each customer so as to minimize total expected costs, comprising costs of earliness, lateness, product shortfall, and returning to depot non-empty. This problem can be encountered within an "industrial gases context".

Following the above classification, our work can be included in the Finite Horizon and multi-period group. The model has infinite capacity at the warehouse, distributor faces both stockout and holding costs, initial stock is only known at the beginning of the first period and each customer is visited at least once in the planning period. This model distances from the above literature on another aspect: the incorporation in the routing problem of both CMI points and VMI points.

\section{The MPIRP-SDD}

In this MPIRP-SDD model we consider two types of customers: the VendorManaged Inventory (VMI) customers and the Customer Managed Inventory (CMI) customers. The VMI customers have a random demand with a known distribution function for each period in the planning horizon. The distributor manages the stock at the VMI customer location and is responsible for the inventory cost incurred on these locations. For this group, it is the responsibility of the distributor to decide how much to deliver and when. The distributor has two types of costs related to these points: the holding cost ( i.e. the cost of having inventory at these points, this cost is per unit and per period); and a stock out cost (i.e. cost per unit not sold).

The CMI have fixed demand that has to be fully satisfied on a specific day and the distributor faces no inventory costs associated with these customers. The CMI customers place an order to the distributor, some time in advance, to be delivered on an agreed date. These customers decide the quantity and

\footnotetext{
${ }^{5}$ Fixed Partitioning Policies, partitions the set of retailers into a number of regions, such that each region is served separatly and independently from all other regions, whenever a retailer in a set is visited by a vehicle, all other retailers in the set are visited as well.
} 
delivery days and the distributor has no responsibility on the inventory they posses.

The objective is to determine the routes for a week planning period (composed of five working days) and the quantities delivered at the VMI points, minimizing total transportation plus inventory costs. The choice of a week planning period is motivated by two aspects: the strategic perspective of the model, our objective is to design a distribution policy in advance; and for control reasons, there is a minimum frequency of visiting a customer, we assume that it is at least once in the planning period. We have to decide when to visit the VMI customers and how much to deliver each time we visit them. The costs of the model include traveling cost, inventory managing costs associated with the VMI customers and a fixed cost of using a vehicle.

Although there exist some literature on the integration issue, none of them addresses the specific case of this model: two types of customers, a week minimum visit and no information on the inventory levels during the period.

The motivation of this work is based on the need for coordination within the supply chain management. In this case, we are trying to coordinate decisions from the distribution management area with decisions from the inventory management area. The aim of this model is, by coordinating strategies in different areas of the SCM try to reduce total costs. In other words, define an integrated inventory-routing strategy that proves to be more efficient than a non integrated inventory routing strategy (solving both problems independently).

So, based on this idea, the objective of this model will be to design the routes and the delivering quantities in such a way has to minimize total cost. The decisions will be based on the assumptions explained below.

\subsection{Assumptions of the model}

The model tries to define the best routes for all customers and the best delivering quantities for the VMI customers. The first assumption is that we have two sets of customers and the VMI customers are visited at least once a week. Another important aspect is that we only know the initial inventory at the beginning of the first period, and the decisions are taken for the all week, independently of what occurs during the week.

The assumptions of the model are.

- Set of customers with well known geographical locations.

- Week period delivery system is considered (five working days). 
- The CMI customers have a demand that is fixed, that is, at the beginning of the period we know the demand and, this exact amount has to be delivered at a specific day.

- There are no inventory costs to be managed at the CMI sites.

- There are no handling stock costs at the depot, and we assume that there is enough amount of product at the depot (unlimited capacity).

- VMI customers have stochastic demand, the quantities to deliver depend on the expected demand.

- For the VMI customers, demand probability function is known and varies by customer and from day to day.

- At the VMI points, there are inventory holding costs and a cost for stock out.

- The vehicles have a fixed capacity.

- The highest demand is always smaller than a vehicle capacity.

- There is no fixed number of vehicles but a high fixed cost for the use of a vehicle.

- Each customer can only be visited once a day and is visited at least once a week.

- The holding and stock out costs only depend on quantities and not on customers. And the stock out cost is always bigger than the holding cost.

\subsection{The MPIRP-SDD}

\section{The costs of the problem are:}

- Transportation cost between locations;

- Stock out and inventory costs for the VMI customers, per unit of product and per day;

- Fixed cost per vehicle used.

The decisions to be made are the following: 
- Decide the routes for each day of the week, for each vehicle;

- Decide the number of vehicles needed each day of the period;

- Decide for each day which of the VMI customers will be included and where in the tour;

- How much to deliver to the VMI customers, on each day.

\section{Objective function:}

Objective is to minimize the expected cost at the end of the week:

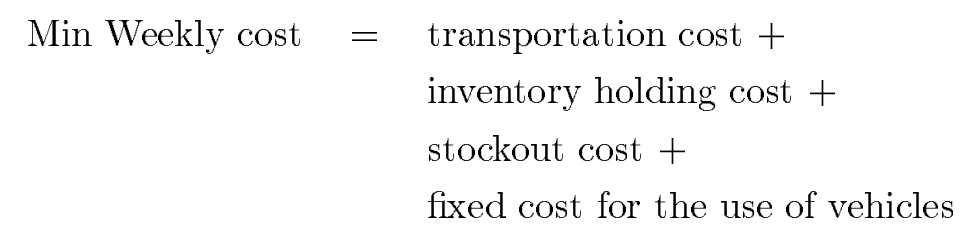

\section{Notation:}

$A=$ set of CMI customers.

$B=$ set of VMI customers.

$n=$ number of customers, indexed from 1 to $n$; index 0 denotes the central depot.

$Q=$ capacity of a vehicle.

$P=$ number of periods (in this case, $P=5$ periods, from 0 to 4 ).

$c_{i j}=$ cost of direct travel from location $i$ to location $j$.

$F_{i p}()=$. cumulative distribution function of the demand of one period, in location $i$, with $i \in B$ for day $p$.

$f_{i p}=$ distribution function of the one period demand for customer $i$ and day $p, i=1, \ldots n$ and $p=1, \ldots, P$;

$h=$ inventory holding cost per unit and per day.

$s=$ shortage cost per unit and per day.

$\beta_{i}^{p}=$ initial inventory at location $i$ on day $p$. 
$d_{i}^{p}=$ demand of customer $i$ on day $p$ with $i \in A$.

$T_{i}=$ set of days where $i$ has a positive demand, with $i \in A$.

$C=$ fixed cost per vehicle used per day.

$K=$ maximum number of available vehicles.

\section{Variables:}

$x_{i j k}^{p}= \begin{cases}1, & \text { if vehicle } k \text { travels directly } \\ 0, & \text { otherwise } .\end{cases}$

$y_{i k}^{p}= \begin{cases}1, & \text { if customer } i \text { is assigned to vehicle } k \text { on day } p \\ 0, & \text { otherwise. }\end{cases}$

$w_{i k}^{p}=$ amount delivered to location $i$ on day $p$, by vehicle $k$.

$$
K_{p}=\text { number of vehicles needed on day } p .
$$

\section{Transportation cost:}

$$
\sum_{i, j, k} c_{i j} x_{i j k}^{p}
$$

\section{Inventory cost:}

Consider

$$
\begin{gathered}
w_{i}^{p}=\sum_{k=1}^{K} w_{i k}^{p} \\
w_{i}^{p}=\text { amount delivered to location } i \text { on day } p \\
\sum_{i \in B} \sum_{p=\mathbf{0}}^{P-1}\left[h \sum_{\mathbf{0}}^{\beta_{i}^{p}+w_{i}^{p}}\left(\beta_{i}^{p}+w_{i}^{p}-t_{i p}\right) f_{i p}\left(t_{i p}\right) d t_{i p}+s \sum_{\beta_{i}^{p}+w_{i p}}^{\infty}\left(t_{i p}-\beta_{i}^{p}-w_{i}^{p}\right) f_{i p}\left(t_{i p}\right) d t_{i p}\right]
\end{gathered}
$$

Fixed cost per vehicle used: 


$$
C \times \sum_{p=0}^{P-1} K_{p}
$$

Let $I_{i p}\left(w_{i}^{p}\right)$ represent the inventory managing cost of VMI location $i$ on day $p$.

The problem can be stated as follows:

$\operatorname{Min} \sum_{i=\mathbf{0}}^{n} \sum_{j=\mathbf{0}}^{n} \sum_{k=1}^{K} \sum_{p=\mathbf{0}}^{P=1} c_{i j} x_{i j k}^{p}+\sum_{i \in B} \sum_{p=\mathbf{0}}^{P=1} I_{i p}\left(w_{i}^{p}\right)+\sum_{p=\mathbf{0}}^{P=1} K_{p} * C$

Subject to:

$$
\begin{aligned}
& \sum_{k=1}^{K} y_{i k}^{p}=1, \quad \forall i \in A, \quad \forall p \in T_{i} \\
& \sum_{k=1}^{K_{p}} y_{0 k}^{p}=K_{p}, \quad \forall p=0, \ldots, P-1 \\
& \sum_{i \in A} d_{i}^{p} y_{i k}^{p}+\sum_{i \in B} w_{i k}^{p} \leq Q, \quad \forall p=0, \ldots, P-1 \quad k=0, \ldots, K \\
& \sum_{j=0}^{n} x_{i j k}^{p}=\sum_{j=0}^{n} x_{j i k}^{p}=y_{i k}^{p}, \forall i=1, \ldots, n ; k=1, \ldots, K_{p} ; p=0, \ldots, P-1 \\
& \sum_{j, i \in S} x_{i j k}^{p} \leq|S|-1, \forall S \text { nonempty subset of }\{1, \ldots, n\} \\
& k=1, \ldots, K ; p=0, \ldots, P-1 \\
& 1 \leq \sum_{k=1}^{K} \sum_{p=0}^{P-1} y_{i k}^{p}, \quad \forall i \in B \quad \forall \\
& \sum_{k=1}^{K} y_{i k}^{p} \leq 1, \quad \forall i \in B \quad p=0, \ldots, P-1 \\
& y_{i k}^{p} \leq w_{i k}^{p} \leq y_{i k}^{p} M, \quad \forall i \in B \quad p=0, \ldots, P-1 ; \quad k=1, \ldots, K ; \\
& w_{i k}^{p} \geq 0, \quad p=0, \ldots, P-1 \\
& x_{i j k}^{p} \in\{0,1\} ; y_{i k}^{p} \in\{0,1\} \forall i=0, \ldots, n ; k=1, \ldots, K ; p=0, \ldots, P-1
\end{aligned}
$$

The meaning of the above constraints is: 
(2) For the CMI, in days where the customer have a positive demand, that customer is visited by only one vehicle.

(3) The second constraint forces that each day all vehicles go to the depot.

(4) This constraint ensures that the daily loading of a vehicle does not exceed its capacity.

(5) This constraint guarantees that if the vehicle enters a node, on day $p$, it also has to leave that node, on the same day.

(6) Avoids sub-tours, for each vehicle for each day. The sub-tour elimination constraint represents an exponential number of constraints. The problem with this constraint is that its number grows exponentially with $n$.

(7) The VMI customers are visited at least once a week.

(8) Each day, there can only be at most one vehicle visiting the VMI customers.

(9) If the amount delivered, by vehicle $k$, to a VMI $i$ on day $p$ is positive then, vehicle $k$ has to visit that location on that day. $M$ represents a very big value.

(10) The variable $w_{i p}$, representing the quantity delivered to a random demand customer on a given day is always greater or equal to 0 .

(11) This constraint define the variables $x_{i j k}^{p}$ and $y_{i k}^{p}$ as binary.

The inventory cost only applies for the VMI customers and is equal to: the expected inventory holding cost, if the initial stock plus the quantity delivered is less than the demand, or a stockout cost otherwise. This expression applies for the case where demand is a discrete variable.

Since the representation of the probability distribution is difficult to find, particularly when demand ranges over a large number of possible values, the discrete random variable is often approximated by a continuous random variable. Furthermore, when demand ranges from over a large number of possible values, this approximation will generally yield a nearly exact value of the optimal amount. In addition, when discrete demand is used, the resulting expression becomes more difficult to solve analytically.

So, from now on we will consider the demand of the VMI customers as a continuous random variable: 


$$
\begin{aligned}
& \sum_{i \in B} \sum_{p=\mathbf{0}}^{P-1} I_{i p}\left(w_{i}^{p}\right) \\
= & \sum_{i} \sum_{p}\left[\begin{array}{c}
h \int_{0}^{\beta_{i}^{p}+w_{i}^{p}}\left(\beta_{i}^{p}+w_{i}^{p}-t\right) f_{i p}(t) d t \\
+s \int_{\beta_{i}^{p}+w_{i}^{p}}^{\infty}\left(t-\beta_{i}^{p}-w_{i}^{p}\right) f_{i p}(t) d t
\end{array}\right]
\end{aligned}
$$

The above inventory cost function (12) only works if the initial inventory of each period is known in advance, or observed. Since we are planning for several periods in advance we do not know the demand on each period. The initial inventory is then also a random variable that depends on the demand of all the previous periods and on quantities delivered in all the previous periods.

We will assume that the only quantity we can observe is the initial inventory of each customer at the beginning of period 0 .

At each period, if there is a stockout, a cost is incurred and the initial stock of the following period will be zero.

Then, the initial inventory for each period is:

$$
\begin{aligned}
& \beta_{i 0}=u_{i}>0 \\
& \beta_{i 1}=\operatorname{Max}\left\{0, u_{i}+w_{i 0}-t_{i 0}\right\} \\
& \beta_{i 2}=\operatorname{Max}\left\{0, \beta_{i 1}+w_{i 1}-t_{i 1}\right\}= \\
& \operatorname{Max}\left\{0, \operatorname{Max}\left\{0, u_{i}+w_{i 0}-t_{i 0}\right\}+w_{i 1}-t_{i 1}\right\} \\
& \beta_{i 3}=\operatorname{Max}\left\{0, \beta_{i 2}+w_{i 2}-t_{i 2}\right\}= \\
& \operatorname{Max}\left\{0, \operatorname{Max}\left\{\begin{array}{c}
0, \operatorname{Max}\left\{0, u_{i}+w_{i 0}-t_{i 0}\right\}+ \\
w_{i 1}-t_{i 1}
\end{array}\right\}+w_{i 2}-t_{i 2}\right\} \\
& \beta_{i 4}=\operatorname{Max}\left\{0, \beta_{i 3}+w_{i 3}-t_{i 3}\right\}=
\end{aligned}
$$

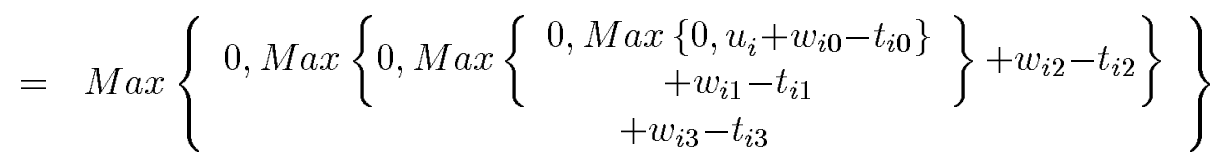

So, the initial stock of period $p$ will be a function of the initial stock of period 0 , of the quantities delivered and demand of all previous periods.

$$
\beta_{i}^{p}\left(u_{i}, w_{i 0}, \ldots, w_{i p-1}, t_{i 0}, \ldots t_{i p-1}\right)
$$


Assume from now on that the demand follows an exponential distribution function with the form:

$$
f(t)=a_{i} e^{-a_{i} t}
$$

There are many different statistical distribtuions to choose from when working with inventory control. The exponential distribution function is commonly used to represent the demand variation in inventory models. See Lidke and Malstrom (1987) and Snyder (1984).

We need to calculate the inventory cost for each period. So, for each period we need to consider all the possible scenarios of the previous periods initial inventory. This is, for period 0 , we know the initial inventory, for period 1 we need to consider two possibilities: stock-out at the end of period zero (zero initial inventory at period 1) and positive stock at the end of period 0 (initial stock at period one positive). Then we extend this for the second, third and fourth periods for all possible combinations.

The expression of the inventory cost function for each customer and each period can be seen in detail in Ribeiro and Lourenço (2003).

\section{Heuristics Solution Method for the MPIRP-SDD}

The limitations of available computational techniques make it impractical to try to solve this problem directly for all but very small instances. The structure of the problem argues for some type of decomposition. As mentioned there are two subproblems embedded in the IRP. The first is to decide the delivery day and the quantity, the second involves routing decisions.

Our decomposition scheme for the IRP is outlined in the following steps:

- Step 1: Obtain an initial solution where the inventory problem is solved separately without considering any delivery cost.

- Step 2: For each day in the planning horizon, try to find a good feasible solution by solving a VRP.

- Step 3: Calculate an approximation of the VMI customers delivery cost.

- Step 4: Determine the new quantities and delivery days for the VMI customers, taking into account the setup cost. 
- Step 5: For each day in the planning horizon, try to find a good feasible solution by solving a VRP.

- Step 6: Repeat steps 3 and 4 until a satisfied solutions is found.

Next, we will explain in more detail each step of the heuristics for the MPIRP-SDD.

\section{Step 1: The Initial solution - The inventory problem}

In the first step of the algorithm we will solve the inventory problem alone. Considering that there are no transportation costs to be handled. The cost function (12) only works if the initial inventory of each period is known in advance. However, one of the assumptions of the model is that the stock at the VMI locations can only be observed at the beginning of the planning period. Therefore, the initial inventory is also a random variable that depends on the demand of all the previous periods and on quantities delivered in all the previous periods.

We need to calculate, for each VMI customer, the inventory cost for each period.

For period zero, we observe the initial stock and incur in holding cost if expected demand exceeds the initial inventory plus the quantity delivered or a stockout cost otherwise. If there is a stockout, in the next period the initial stock will be zero.

For the other periods (period 1 to 4), we have to consider all the possible scenarios of the previous periods. For period 1, for example, we need to consider two possibilities: stock-out in period zero (zero initial inventory at period 1) and positive stock at the end of period 0 (initial stock at period one positive). For each VMI customer $i$ :

Period 1: $\left\{\begin{array}{l}\beta_{1}=0 \\ \beta_{1}>0\end{array}\right.$

For period 2 we need to consider 4 scenarios:

Period 2: $\left\{\begin{array}{l}\beta_{1}=0 \text { and } \beta_{2}=0 \\ \beta_{1}=0 \text { and } \beta_{2}>0 \\ \beta_{1}>0 \text { and } \beta_{2}=0 \\ \beta_{1}>0 \text { and } \beta_{2}>0\end{array}\right.$

Then we extend this for period 3 and 4 following the same reasoning. 
This inventory problem, is different from other inventory problems that can be found in the literature. Since, in this particular case we have a planning horizon, 5 days, for which we need to plan the delivery quantities. Also, we do not known what is the initial stock at each day for each customer. We have to decide how often and how much to deliver. Since we do not allow for negative quantities, we have a non-linear constrained minimization problem.

This initial solution is obtained by solving the inventory model for each customer. The inventory cost function results in a complex integral with 5 integrals. This expression is minimizes using the Gauss-Newton method, with the average demand as a starting point. The objective is to minimize inventory costs (holding + stock-out costs). The best solution is to deliver almost every day to the VMI customers, since there is no setup cost associated with the deliveries.

In summary, in step 1 we calculate the optimal quantities to deliver on each day of the week to each VMI customer. We assume an initial solution for the first day and an exponential distribution function for the demand of each customer on each day. For the detailed inventory cost function analysis and solution method see Ribeiro and Lourenço (2003).

\section{Step 2: The VRP}

After step 1, we known the quantities and the days to delivery to each customer. Therefore, we have to solve a VRP each day to obtain the best routes. To solve the VRP each day, we consider an ILS for all customers on that specific day and their respective quantities. After, we calculate the total cost: transportation cost and inventory cost associated with the VMI customers.

Due to the complexity of the VRP problems (NP-hard) we need to develop an heuristic. Our proposal is to use an heuristic algorithm that as proven to give quiet good results on other problems and is easy to implement and modify.

A heuristic algorithm is a solution method that does not guarantee an optimal solution, but in general has a good level of performance in term of solution quality and convergence. Heuristics may be constructive (producing a single solution) or local search (starting from one given random solution and moving iteratively to other nearby solutions) or combination of both.

Iterated Local Search (ILS) is a simple and generally applicable Metaheuristic which iteratively applies local search to modifications of the current search point. At the beginning of the algorithm, a local search is applied to 
some initial solution. Then, a main loop is repeated until a stopping criterion is satisfied. This main loop consists of a modification step ("perturbation"), which returns an intermediate solution corresponding to a modification of a previously found locally optimal solution. Next, local search is applied to yielding a locally optimal solution. An "acceptance criterion" then decides from which solution the search is continued by applying the next "perturbation". Both, the perturbation step and the acceptance test may be influenced by the search history. ILS is expected to perform better than if the method restarts local search from a new randomly generated solutions.

ILS algorithms have been applied successfully to a variety of combinatorial optimization problems. In some cases, these algorithms achieve extremely high performance and even constitute the current state-of-the-art metaheuristics, while in other cases the ILS approach is merely competitive with other metaheuristics. ILS has many of the desirable features of a metaheuristic: it is simple, easy to implement, robust, and highly effective. For a survey in ILS see Lourenço, Martin and Stützle (2001) and Lourenço, Martin and Stützle (2002).

Here is a structure of the ILS:

procedure ILS

$s^{0}=$ GenerateInitialSolution

$s^{*}=\operatorname{LocalSearch}\left(s^{0}\right)$

repeat

$s^{\prime}=\operatorname{perturbation}\left(s^{*}\right.$, history $)$

$s^{* \prime}=$ Local $\operatorname{search}\left(s^{\prime}\right)$

$s^{*}=$ AcceptanceCriterion $\left(s^{*}, s^{* \prime}\right.$, history $)$

until termination condition met

end

The ILS used was based in the heuristic developed by Stützle(1998) and Kunz (2000). The structure of the algorithm is the following:

Savings heuristic (Clarke and Wright (1964)) for the initial solution.

\section{ILS for TSP on each tour:}

- Local Search for TSP: 2-opt.

- Kick move for TSP: double bridge, this perturbations cuts four edges, and introduces four new ones.

- Acceptance criterion: better; this means that the new tour is accepted if it has a lower cost. 


\section{ILS for the VRP}

- Local Search for the assignment problem: 2-opt. We have two possibilities for a 2-opt: A customer of a tour is postponed into another or a customer trades with another customer from another tour. First, if capacity restrictions allow and it reduces costs, a city is inserted in the tour. Only if it can not be inserted, then we check if an exchange with another tour improves the solution.

- Kick move for VRP: Numb-crosser: exchanges final pieces through the number of customers, exchanges $1 / 3$ of the tour, a tour is chosen by chance and then break the tour.

- Acceptance criterion: Best;

ILS for the TSP on the new routes: The same as for the first ILS implemented in the first part.

At the end of 2, we have obtained an initial solution for the MPIRPSDD model based on a sub-optimization of the two existing problems: the routing and the inventory problem. Now, Step 3 to 6 consists in developing an heuristic method that improves this initial solution.

\section{Step 3: Inventory and transportation - The setup cost approach}

When considering the inventory problem separately from the transportation problem, the best solution is to deliver frequently, every day or almost every day, however, this implies higher transportation costs. Our objective is to balance the delivery costs with the inventory cost. One way to do this is by considering a setup cost: a cost per delivery made to a VMI customer. This setup cost only applies to these set of customers since, the CMI customers have to be visited on a specified day and no changes are allowed on these customer's orders. If a VMI customer is visited on day $p$, then there is a fixed cost associated with this customer, on this day.

We will obtain the setup cost by calculating the approximate cost of serving a VMI customer on a specific day, this is, the cost of including a customer $i$ on tour $j$ on day $p$. This is an approximation since the real value would have to consider the relationship between the delivering decisions of all the other customers and the best corresponding routes.

It is logical to think that if a customer is close to a group of customers that are visited on day $p$, than we have a lower cost of delivering that 
customer on that day. This is, the setup cost of customer $i$ will depend on the location of this customer with respect to other customers in the route.

This cost represents the cost associated with delivering to a customer on a day $p$, and will only depend on distance. This setup cost will be calculated in the following way:

$g_{i}^{p}=$ cost of going to customer $i$ on day $p$.

$\theta=$ a fixed setup weight per distance unit.

Let $j$ and $k$ be the previous and successor customer in the route of customer $i$ on day $p$, then.

$$
g_{i}^{p}=\left(c_{j i}+c_{i k}-c_{j k}\right) \times \theta
$$

At Step 3 we have calculated the setup cost for each VMI customer on each day.

\section{Step 4: New deliveries}

Now, we have an inventory model with a setup cost. For each VMI customer we need to redefine the optimal deliveries. For a more detail discription of this inventory model with setup costs see Ribeiro and Lourenço(2003).

The procedure can be summarized as follows:

- Consider all possible combinations of delivery days.

Solve the inventory problem for each combination.

Add the corresponding setup cost in each solution.(obtained in Step 3)

Choose, for each VMI customer, the solution with the lowest total cost (inventory + setup).

At this stage we obtain the new set of delivery days and quantities.

\section{Step 5: New VRP}

For each day in the planning horizon, and using the new delivering quantities from Step 4, find a good feasible solution by solving a VRP (repeating Step 2).

\section{Step 6: Repeat}


Repeat steps 3 to 6 until a satisfied solution is found, or until a certain number of iterations have been performed.

We will present a computational experiment on the application of this method to the MPIRP-SDD.

\section{Computational Results}

In this section we will present a computational study that assess the impact of integrating transportation and inventory. To analyse this impact it is interesting to compare two solutions: The integrated solution and the non-integrated solution. This non-integrated solution is characterized by the separability of the two problems: the inventory and the routing are independent, first we solve the inventory problem and use the solution to decide the routes. The solution of the integrated problem is the one obtained after the running of the algorithm presented in the previous section.

The objective is to compare the two solutions (the integrated versus the non-integrated solution) obtained when solving the two different problems (Inventory and Routing) and analyzing the impact of considering the problems in an integrated form.

There are two ways in which we will orient this analysis: the first is to compare total costs; and the second is to make a multi-objective approach. In this second perspective the inventory cost and the routing cost are the two objectives that we would like to minimize. Instead of choosing the best total cost we will consider all non-dominated solutions obtained from the algorithm and would be the responsibility of the decision maker to choose.

Next we will explain the data used and analyze some important results of this experiment.

\section{$5.1 \quad$ The data}

For the computational experiment we have generated several sets of examples, each group with different characteristics concerning: total number of customers $(100,200,400)$; percentage of VMI customers (10\% and 50\%); type of demand (equal every day, different each day) and setup cost parameter (high setup cost $\theta=100$ per distance unit and low setup cost, $\theta=10$ per distance unit).

For the VMI customers, we have used a demand parameter $\alpha_{i p}$ (for each customer $i$ and day $p$ ) that follows a normal distribution with mean 50 and 
standard deviation 20 for each customer. In the cases where demand is different every day the standard deviation between days used was 5 .

The initial stock for each customer was generated by a random uniform distribution between 0 and 50 .

The results were obtained considering 8 iterations. The stockout cost used was twice the holding cost, in this experiment $h=2$ and $s=4$. There is a fixed charge per vehicle used per day: $C=200$.

\subsection{Analysis of the results}

We can start by looking at a few examples in terms of the solutions obtained at each iteration, see Figure 1.

The non-integrated solution corresponds to the initial solution obtained at the end of step 2 of the algorithm. By continuing the algorithm (step 3 to 6) and exploring other VMI delivery strategies, the new inventory cost increases but, allows some savings in terms of routing. In Figure 1, iteration 0 corresponds to the non-integrated solution while the other iterations 1 to 7 correspond to integrated solutions. In these two particular examples, the best solution would be found at iterations 1 . However, supposing that a higher preference was given to reduce routing costs, then the best solution would be at iteration 7 for example $\mathrm{A}$ and 2 for example B. 
Example A: Inventory, Routing and Total Costs, example Example B: Inventory, Routing and Total Costs, example of 100 customers, $50 \%$ VMI, low setup cost.

of 200 customers, $10 \% \mathrm{VMI}$, high setup cost.
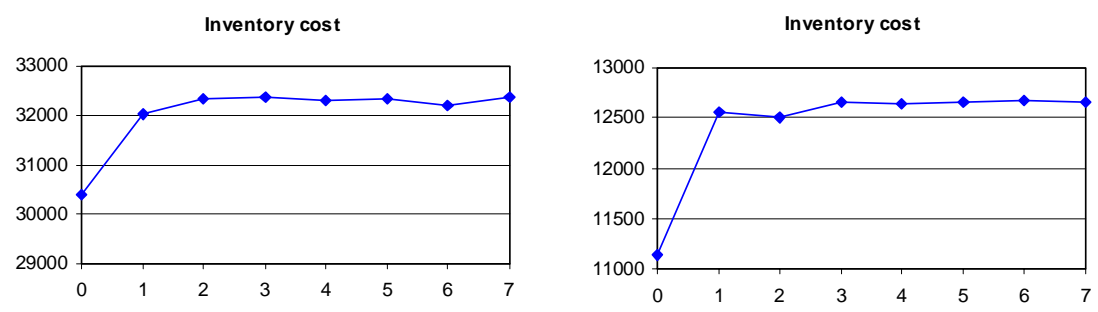

Transportation cost

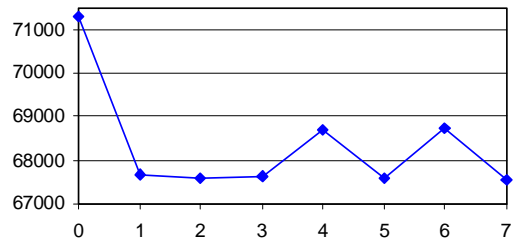

Transportation cost

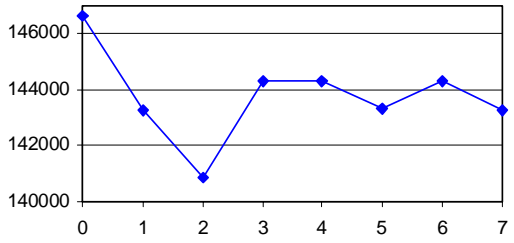

Total cost

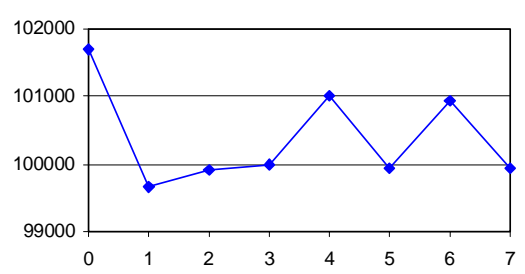

Total cost

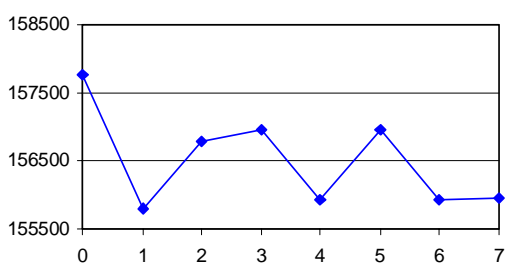

Figure 1: Examples of Inventory, Routing and Total Costs at each iteration.

Figure 1: Examples of Inventory, Routing and Total Costs at each iteration.

In Table 1, we present the average total cost improvemet for each problem size. This average Cost Reduction was calculated by comparing, for each group of examples, the best solution for the non-integrated (i.e. the initial solution obtained at the end of step 2) versus integrated case (i.e. the best solution in terms of total cost, obtained by the algorithm). The average savings, for instances of 100 customers with $10 \%$ VMI customers, when integrating routing and inventory is $1,42 \%$ of the total cost. 


\begin{tabular}{rrc}
\hline $\begin{array}{r}\text { Problem } \\
\text { Size }\end{array}$ & $\begin{array}{r}\text { Number } \\
\text { VMI }\end{array}$ & $\begin{array}{r}\text { Average Total Cost } \\
\text { Reduction }\end{array}$ \\
\hline \hline 100 & 10 & $1,42 \%$ \\
& 50 & $0,99 \%$ \\
\hline 200 & 20 & $0,94 \%$ \\
& 100 & $2,09 \%$ \\
\hline 400 & 40 & $0,26 \%$ \\
& 200 & $0,06 \%$ \\
\hline
\end{tabular}

Table 1: Average Total Cost Reduction for each group size.

In Figure 2, we can see the trade-off between Inventory cost and Routing cost. This corresponds to viewing the problem has a multi-objective problem, where all the set of non-dominated solution are of interest for the decision maker to choose the best delivering and routing strategy. In example $C$ we would have three non-dominated solutions (the three circles in the graph).In example $D$, only two solutions would be non-dominated (the two circles in the graph).
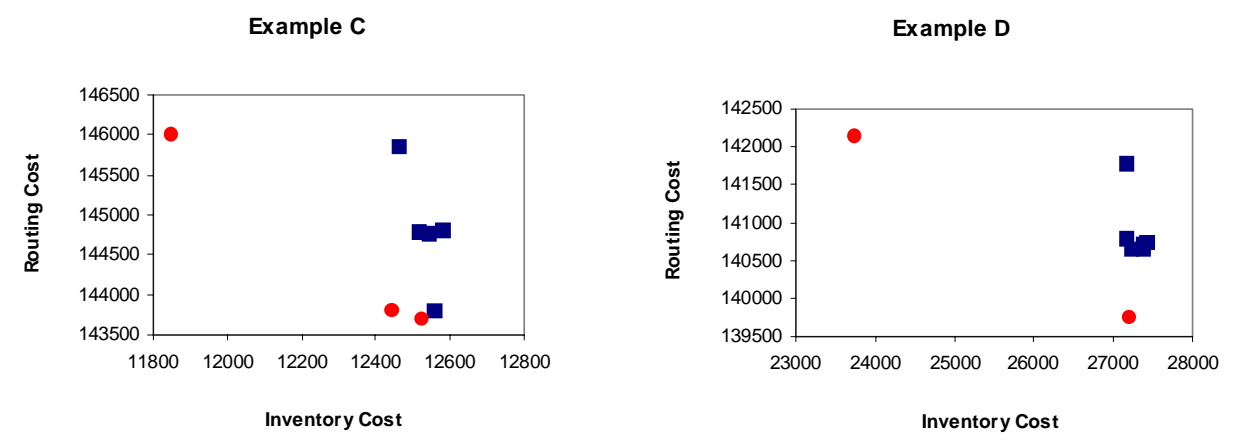

Figure 2: Trade-off between Inventory and Transportation cost.

We also compared the non-integrated solution with the best solution in terms of routing cost. Table 2 considers the trade-off between inventory and routing costs when going from the solution of the non-integrated case 
to the integrated one. The results are shown in terms of average variation in routing and inventory: the inventory costs increase and the routing costs decrease.

The best solution in terms of transportation cost always implies an increase in inventory cost. The difference in the magnitude of these variations is justified on one hand by the values chosen for the inventory costs and transportation cost. And, on the other hand, by the existence of other customers, the CMI customers. These customers have fixed delivery days and quantities: The higher is the percentage of CMI customers the less we can reduce routing costs by concentrating deliveries (the impact on total routing cost is less visible).

\begin{tabular}{|c|c|c|c|c|c|c|c|c|c|}
\hline \multirow{3}{*}{$\begin{array}{l}\text { Problem } \\
\text { Size }\end{array}$} & \multirow{3}{*}{$\begin{array}{l}\text { Number } \\
\text { VMI }\end{array}$} & \multicolumn{4}{|c|}{ High setup cost parameter } & \multicolumn{4}{|c|}{ Low setup cost parameter } \\
\hline & & \multicolumn{2}{|c|}{ Equal demand } & \multicolumn{2}{|c|}{ Different demand } & \multicolumn{2}{|c|}{ Equal demand } & \multicolumn{2}{|c|}{ Different demand } \\
\hline & & ip_cost & vrp_cost & ip_cost & vrp_cost & ip_cost & vrp_cost & ip_cost & vrp_cost \\
\hline \multirow[t]{2}{*}{100} & 10 & $-0,19$ & 0,03 & $-0,22$ & 0,03 & $-0,07$ & 0,01 & $-0,08$ & 0,02 \\
\hline & 50 & $-0,21$ & 0,05 & $-0,19$ & 0,04 & $-0,06$ & 0,05 & $-0,06$ & 0,05 \\
\hline \multirow[t]{2}{*}{200} & 20 & $-0,16$ & 0,03 & $-0,15$ & 0,02 & $-0,05$ & 0,01 & $-0,03$ & 0,01 \\
\hline & 100 & $-0,18$ & 0,12 & $-0,17$ & 0,12 & $-0,04$ & 0,03 & $-0,04$ & 0,03 \\
\hline \multirow[t]{2}{*}{400} & 40 & $-0,15$ & 0,02 & $-0,14$ & 0,02 & $-0,02$ & 0,01 & $-0,02$ & 0,01 \\
\hline & 200 & $-0,15$ & 0,11 & $-0,15$ & 0,11 & $-0,03$ & 0,02 & $-0,02$ & 0,02 \\
\hline
\end{tabular}

Table 2: Trade-off between inventory and routing costs.

In terms of delivery days, when optimizing separately we obtain an average of 4,68 delivery days a week, for the VMI customers, while for the integrated case, this average falls to 3,49 delivery days a week.

Another analysis that can be done is in terms of vehicles needed. When we integrate transportation and inventory, this implies that the delivery frequency is reduced and we are able to reduce the number of routes. Assuming that the distributor pays a fixed charge per use of a vehicle then, by reducing the number of routes at the end of the week the distributor is able to reduce routing costs. Table 3 , shows for each group of examples, the average reduction in the number of vehicles needed per week. The higher is the percentage of VMI customers, the more we can reduce the number of vehicles needed. This reduction is higher when the setup cost parameter is high. A higher setup cost parameter means that solutions with fewer deliveries are preferable. For example, in the group with 100 customers 10 of them VMI, for the integrated solution, the total number of vehicles needed per week reduces on average $2,81 \%$ and $1,73 \%$ for the cases with high and low setup cost parameter respectively. 


\begin{tabular}{llcc}
\hline $\begin{array}{l}\text { Problem } \\
\text { Size }\end{array}$ & Number & VMI & \multicolumn{2}{c}{ Reduction N. of vehicles } \\
\cline { 3 - 4 } & 10 & $\begin{array}{c}\text { High setup cost } \\
\text { parameter }\end{array}$ & $\begin{array}{c}\text { Low setup cost } \\
\text { parameter }\end{array}$ \\
\hline \hline 100 & $-2,81 \%$ & $-1,73 \%$ \\
& 50 & $-12,34 \%$ & $-4,67 \%$ \\
\hline 200 & 20 & $-2,55 \%$ & $-1,65 \%$ \\
& 100 & $-12,02 \%$ & $-3,19 \%$ \\
\hline 400 & 40 & $-2,08 \%$ & $-0,99 \%$ \\
& 200 & $-10,65 \%$ & $-2,14 \%$ \\
\hline
\end{tabular}

Table 3: Reduction in the total number of vehicles.

In terms of run time, Table 4 summarizes the average number of running time per problem, measured in seconds:

\begin{tabular}{llc}
\hline $\begin{array}{l}\text { Problem } \\
\text { Size }\end{array}$ & $\begin{array}{l}\text { Number } \\
\text { VMI }\end{array}$ & $\begin{array}{c}\text { Average Run } \\
\text { Time in seconds }\end{array}$ \\
\hline \hline 100 & 10 & 110,73 \\
& 50 & 113,88 \\
\hline 200 & 20 & 465,72 \\
& 100 & 376,39 \\
\hline 400 & 40 & 1783,901 \\
& 200 & 1490,591 \\
\hline
\end{tabular}

Table 4: Average run time

The non-integrated inventory solution consists of delivering almost every day. In Table 5, we see the average number of delivery days, for the nonintegrated problem for examples with different variations in demand. For each group the standard deviation of demand between days is $0,5,10,20$. The higher the standard deviation, the fewer the average number of delivery days. 


\begin{tabular}{|c|c|}
\hline \multicolumn{2}{|c|}{ Average number of delivery days } \\
\hline $\begin{array}{c}\text { Demand } \\
\text { variations } \\
\end{array}$ & $\begin{array}{c}\text { Inventory } \\
\text { minimization } \\
\end{array}$ \\
\hline 0 & 4,68 \\
\hline 5 & 4,56 \\
\hline 10 & 4,38 \\
\hline 20 & 3,98 \\
\hline
\end{tabular}

Table 5: Average number of delivery days.

The above results show that the distributor who has VMI customers gains from considering inventory and routing in an integrated manner, and this gain can be seen in terms of total cost reduction. The best delivery strategy will be to deliver very frequently which implicates higher transportation costs. When including the cost of delivering, the solution has few delivery days and transportation costs are reduced. The magnitude of this improvement depends on the problem size, on the proportion of VMI customers in the problem and also on the unit costs chosen for both problems. It is also interesting to consider the problem in a multi-objective perspective. For this case, analyze the set of non-dominated solutions and it becomes the responsibility of the decision maker to choose the best solution based on a given inventory strategy and a delivering plan associated.

\section{Conclusions}

The logistic planning functions of transportation and inventory play an important role in may industries and integrating these two areas may lead to significant gains and more competitive distribution strategies. The movement towards more integrated processes cannot ignore these tow key logistic fields.

In this paper, we present a Multi-Period Inventory Routing Problem with Stochastic and Deterministic Demand. We have considered the particular case of a distribution firm that has to decide on its distribution and inventory strategies. This firm has two types of customers, the VMI and CMI customers and decisions have to be made on the quantities delivered and days of visit to the VMI customers, and also in relation with the route planning for the complete set of customers for a week planning period. The 
additional assumption of only observing stock levels at the beginning of the planning period brings more complexity into the model. The objective of this model is to design an integrated Inventory-Routing strategy for a distributor that has to manage inventory and transportation costs of their VMI customers. Considering the inventory and transportation management in an integrated mode can yield to a better performance. As far as we know, there no studies on the IRP with these characteristics. This model can be applied in many distribution processes: for example, in the retailing industry for suppliers of supermarkets and department stores.

An heuristic approach, based on the ILS was constructed to solve this problem. The heuristic has 6 steps, on the first step an initial solution for the inventory problem is obtined. Then, for each day in the planning horizon, tries to find a good feasible solution by solving a VRP. On step 3, an approximation of the VMI customers delivery cost is calculated and based on the results the new quantities and delivery days for the VMI customers are obtained. The process is repeated on the new routes until a satisfied solution is found.

A computational study was done to analyse the impact of integrating Inventory and Routing: the results show that cost reductions are obtained when considering inventory and routing in an integrated manner. The degree of this improvement depends on the problem size, on the proportion of VMI customers in the problem and also on the unit costs chosen for both problems. Given the relationship between the inventory and transportation costs, the decision maker can decide how much of the deliveries to the VMI customers to concentrate.

We are considering future extensions of this work: One includes the analysis of the case where demand faces a distribution function different than the one we have assumed in our work, for example, the Normal distribution. Another interesting extension is to measure the setup costs, in Step 3 of the algorithm, in a dynamic way, this is, taking into consideration not only the precessor and successor customers in the tour but a global effect on the week plan. Finally, we would also like to develop a multi-objective model and solution method to solve the MPIRP-SDD.

\section{References}

[1] Anily, S. and Federgruen, A. (1990). "One warehouse multiple retailer system with vehicle routing costs." Management Science 36(1): 92-114. 
[2] Anily, S. and Federgruen, A. (1991). "Rejoider to "Comments on onewarehouse multiple retailer systems with vehicle routing costs"." Management Science 37(11): 1497-1499.

[3] Anily, S. and Federgruen, A. (1993). "Two-Echelon distribution systems with vehicle routing costs and central inventories." Operations Research 41(1): $37-47$.

[4] Baita, F., Ukovich, W., Pesenti, R. and Favaretto, D. (1998). "Dynamic routing-and-inventory problems: a review." Transportation Research A 32(8): $585-598$.

[5] Bard, J., Huang, L., Jaillet, P. and Dror, M. (1998). "A decomposition approach to the inventory routing problem with satellite facilities." Transportation Science 32(2): 189-203.

[6] Barnes-Schuster, D. and Bassok, Y. (1997). "Direct shipping and the dynamic single-depot/multi-retailer inventory system." European Journal of Operations Research 101: 509-518.

[7] Berman, O. and Larson, R.C. (2001). "Deliveries in an Inventory/Routing Problem Using Stochastic Dynamic Programming." Transportation Science 35(2): 192-213.

[8] Bertazzi, L., Paletta, G. and Speranza, M.G. (2002). "Deterministic order-up-to level policies in an inventory routing problem." Transportation Science 36(1): 119-132.

[9] Burns, L.D., Hall, R.W., Blumenfeld, D.E. and Daganzo, C.F. (1985). "Distribution Strategies that Minimize Transportation and Inventory Costs." Operations Research 33(3): 469-490.

[10] Campbell, A., Clarke, L. and Savelsbergh (1999). "An inventory routing problem." working paper.

[11] Chan, L.M.A., Federgruen, A. and Simchi-Levi, D. (1998). "Probabilistic analysis and practical algorithms for inventory-routing models." Operations Research 46(1): 96-106.

[12] Chien, W., Balakrishnan, A. and Wong, R. (1989). "An integrated inventory allocation and vehicle routing problem." Transportation Science $23(2): 67-76$. 
[13] Clarke, G. and Wright, J., 1964, "Scheduling of vehicles from a central depot to a number of delivery points", Operations Research 12, 568-581.

[14] Dantzing, G.B. and Ramser, J.H., 1959, "The truck dispatching problem", Management Science 6, 80-91.

[15] Dror, M. and Ball, M. (1987). "Inventory/Routing: Reduction from an Annual to a Short-Period Problem." Naval Research Logistics 34: 891-905.

[16] Federgruen, A. and Simchi-Levi, D. (1995). "Analysis of Vehicle Routing and Inventory-Routing Problems". Network Routing. M. O. Ball, Magnanti, T.L., Monma, C.L., Nemhauser, G.L. Amsterdam, Elsevier Science - North Holland. 8: 297-373.

[17] Federgruen, A. and Zipkin, P. (1984). "A combined vehicle routing and inventory allocation problem." Operations Research 32(5): 1019-1037.

[18] Hall, R.W. (1991). "Comments on one warehouse multiple retailer system with vehicle routing costs." Management Science 37(11): 14961497 .

[19] Jaillet, P., Bard, J.F., Huang, L. and Dror, M. (2002). "Delivery costs approximations for inventory routing problems in a Rolling Horizon Framework." Transportation Science 36(3): 292-300.

[20] Kleywegt, A.J., Nori, V.S. and Savelsbergh, M.W.P. (2002). "The Stochastic Inventory Routing Problem with Direct Deliveries." Transportation Science 36(1): 94-118.

[21] Kunz, C. (2000). "Iterierte Lokale Suche ur das Vehicle-Routing Problem", Diplomarbeit (Msc Thesis) TU Damstadt.

[22] Lidke, S. L. and E. M. Malstrom (1987). "A recursive computer algorithm fo determining joint probability inventory distributions." Computers \& Industrial Engineering 12(2): 105-116.

[23] Lourenco, H., Martin, O. and Stützle T. (2001), “A Beginner's Introduction to Iterated Local Search.", In Proceedings of MIC 2001, pages 1-6, Porto, Portugal, July 2001.

[24] Lourenco, H., Martin, O. and Stützle T. (2002), "Iterated Local Search.", In F. Glover and G. Kochenberger, editors, Handbook of Metaheuristics, pages 321-353, Kluwer Academic Publishers, Norwell, MA. 
[25] Ribeiro, R., Lourenço, H. (2003). "Multi-period Vendor Managed Inventory System", working paper, "Mimeo".

[26] Snyder, R. D. (1984). "Inventory control with gamma probability distribution." European Journal of Operational Research 17(3): 373-381.

[27] Stützle, T.G. (1998). "Local Search Algorithms for Combinatorial Problems - Analysis, Improvements, and New Applications". Department of Computer Science, Darmstadt University of Technology.

[28] Trudeau, P. and Dror, M. (1992). "Stochastic Inventory Routing: Route Design with Stockouts and Route Failures." Transportation Science 26(3): 171-184. 